\title{
Tomographic phase space reconstruction during rebucketing in the Relativistic Heavy Ion Collider
}

\author{
C. Montag, N. D’Imperio, J. Kewisch, and R. Lee \\ Brookhaven National Laboratory, Upton, New York 11973
}

(Received 9 July 2002; published 20 August 2002)

\begin{abstract}
Longitudinal phase space tomography has evolved into a powerful diagnostic tool in the particle accelerator domain. A computer code has been developed in order to visualize dynamic effects and measure machine parameters in longitudinal phase space. This code is capable of dealing with turn-by-turn parameter changes, for example, during rf rebucketing when the bunch is rotated in longitudinal phase space to minimize the bunch length. We describe the reconstruction code and show its application as a diagnostic tool for rebucketing in the Relativistic Heavy Ion Collider.
\end{abstract}

DOI: $10.1103 /$ PhysRevSTAB.5.082801

\section{INTRODUCTION}

The basic idea of tomography is to use a sufficiently large number of $(n-1)$-dimensional projections of an $n$-dimensional object to reconstruct the object. For the reconstruction to be feasible, the projections have to be taken from different angles, covering at least $180^{\circ}$ of object rotation.

In the case of a bunch in an accelerator, the necessary rotation is naturally provided by the stable phase space motion of particles in all dimensions. In the framework of this paper, we deal only with longitudinal phase space reconstruction, where projections of the bunch are acquired by a wall current monitor (WCM). In this case, the phase space dynamics involved is not just a simple rotation of a rigid object due to the intrinsic nonlinearity of longitudinal motion in synchrotrons. To overcome this difficulty, the exact particle dynamics has to be taken into account [1]. This is realized by tracking test particles using known machine and rf parameters. During the tomographic reconstruction process, an "intensity" I is iteratively assigned to each test particle according to the measured profiles.

Given a well-defined figure of merit, tomographic reconstruction can also be used to effectively measure rf and/or machine parameters [1]. This is of special interest during processes that involve multiple rf systems and frequent parameter changes, as they occur, for example, during rebucketing.

\section{TOMOGRAPHIC RECONSTRUCTION}

Our tomography code consists of two parts: a tracking routine and an iterative reconstruction algorithm. First, the longitudinal phase space separatrix is calculated according to the initial rf and machine parameters. The area within the separatrix is divided into cells in such a way that the width of each cell in the phase dimension equals the bin width of the measured profiles. The energy span per cell is chosen such that the same number of cells covers the stationary bucket height and the phase between its unstable fixed points. Next, the initial phase space within the sepa-
PACS numbers: 29.20.Dh, 29.27.Eg, 29.27.Fh, 41.85.Ew

ratrix is filled with equidistantly distributed test particles, forming a regular grid. To improve the resolution, each cell is filled with a number of test particles, which are tracked according to the given machine and rf parameters. During tracking, all parameters can be varied on a turn-by-turn basis, allowing reconstruction of rebucketing data.

The test particles are tracked to the time of each measured profile $i$ and are tagged according to their resultant position in the $N_{\text {bins }}$ bins that correspond to the binning of the measured profiles. Thus, each particle $k$ is assigned a bin number $N_{i}^{\text {bin }}(k)$. Additionally, the number of test particles per bin $j$ for the $i$ th profile, $N_{i, j}^{\text {population }}$, is determined and stored.

In the next step, the phase space distribution at the time the first profile was taken is iteratively computed by a simple backprojection reconstruction algorithm. For each profile, $i$, the content $h_{i, j}^{\text {meas }}$ of the $j$ th bin is divided by the number of profiles used for the reconstruction, $N_{\text {profiles }}$, and the number of test particles in the $j$ th bin at the time the $i$ th profile was taken, $N_{i, j}^{\text {population }}$, and added to the intensity $I_{k}$ of each test particle $k$ that could have contributed to the $j$ th bin in the $i$ th profile,

$$
\Delta I_{k \ni N_{i}^{\text {bi }}(k)=j}=\frac{1}{N_{i, j}^{\text {population }} N_{\text {profiles }}} \sum_{i=1}^{N_{\text {profiles }}} h_{i, j}^{\text {meas }} .
$$

This results in a first approximation of the phase space distribution.

In the next iteration step, projected profiles of this reconstructed distribution are calculated as

$$
h_{i, j}^{\text {reconstr }}=\sum_{\forall k \ni N_{i}^{\text {bin }}(k)=j} I_{k},
$$

and the bin-by-bin difference $h_{i, j}^{\text {meas }}-h_{i, j}^{\text {reconstr }}$ is used for backprojection.

After the initial phase space distribution has been determined by sufficient iterations, its evolution can be trivially determined using the same tracking routine as at the beginning. Various tests have been performed to confirm this code. First, the code was applied to artificially generated profiles corresponding to a uniformly populated, circular 

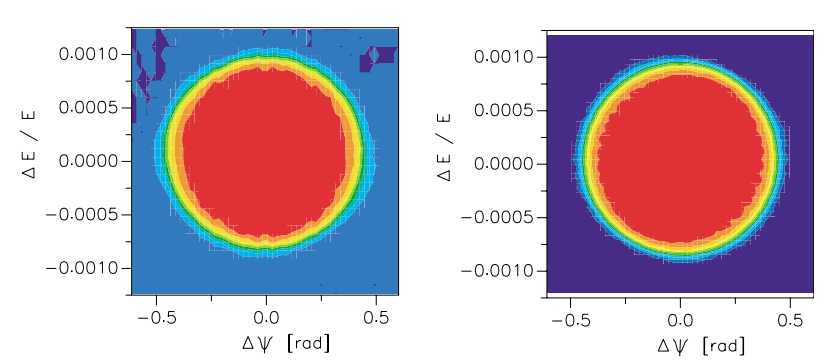

FIG. 1. (Color) Tomographic reconstructions of a circular phase space density distribution using the code described in this paper (left panel) and the CERN code [1] (right panel).
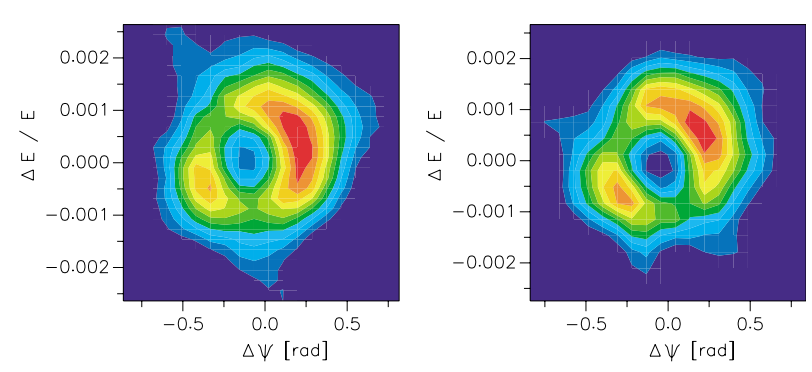

FIG. 2. (Color) Comparison of tomographic reconstructions of a bunch that has been destroyed by a fast instability. The left-hand graph depicts the result obtained by the code described in this paper, while the right-hand one shows the corresponding plot using the CERN code [1].

phase space distribution. Figure 1 shows the result of the tomographic reconstruction. While the flattop reflects the true distribution, the edges are not perfectly sharp but exhibit some slope. However, a comparison with the code described in [1] shows the same result; see Fig. 1. None of the codes is able to reconstruct perfectly, but both come very close.

Applying both codes to real data, only marginal differences occur. Figure 2 shows the reconstructed phase space distribution of a bunch in the RHIC Yellow ring, $0.55 \mathrm{sec}$ after transition crossing. The initially Gaussian shape had been destroyed by a fast instability that set on shortly after crossing transition [2]. This instability lasted only a few turns and left the double-peak bunch structure shown in Fig. 2. Later in the run, octupoles were used to increase Landau damping to fight this instability [3].

\section{RF PARAMETER FITTING}

As mentioned, the difference between the measured profiles and those obtained from the reconstructed distribution is calculated at each iteration. This discrepancy decreases from step to step during the iteration process as the reconstructed distribution approaches the real one. Ideally, the difference between measured and reconstructed profiles vanishes for a sufficient number of iterations if the particle dynamics used in the tracking routine reflects reality. Therefore, this discrepancy, expressed as

$$
\Delta=\sqrt{\sum_{i=1}^{N_{\text {profiles }}} \sum_{j=1}^{N_{\text {bins }}}\left(h_{i, j}^{\text {meas }}-h_{i, j}^{\text {reconstr }}\right)^{2}},
$$

can be utilized as a figure of merit for parameter fitting. Here, $h_{i, j}^{\text {meas }}$ and $h_{i, j}^{\text {reconstr }}$ are, respectively, the measured and reconstructed height of the $j$ th bin in the $i$ th profile.

rf parameter changes in the RHIC are performed by the wave form generator (WFG) manager. Set points are given on a $720 \mathrm{~Hz}$ clock base, corresponding to $f_{\text {rev }} / f_{\text {clock }}=$ $78195 / 720 \approx 108.6$ turns between parameter set point changes. If set points are defined with lower resolution, the WFG manager performs a linear interpolation of these parameters creating additional set points in between those given. Since RHIC rf frequencies of 28 and $197 \mathrm{MHz}$ are high compared to this $720 \mathrm{~Hz}$ clock base, parameter changes occur instantaneously at the respective set point.

Table I shows the rf parameter set points for the so-called phase-back rebucketing procedure in the RHIC. Several seconds after the beams have been ramped to their store energy of $100 \mathrm{GeV} / \mathrm{u}$, the rebucketing procedure is initiated by the rebucketing event. This event triggers data taking by the WCM, which measures the longitudinal profile of a single, selectable bunch every 20 turns. rf parameters are nominally kept at their flattop values for another 890 turns (see Table I). However, since the beginning of data taking is not synchronized with the $720 \mathrm{~Hz}$ clock base, this turn number may vary from fill to fill, leading to some uncertainty of the actual rebucketing time.

On the next turn after the rebucketing event, the phase $\psi_{1}$ of the $28 \mathrm{MHz}$ acceleration cavities is set from its initial value of $\pi$ to zero, moving the center of the bunch to the unstable fixed point where it is kept for $4 \times 108.6$ turns. At turn 1325 the rf phase $\psi_{1}$ is jumped back to $\pi$ and stays there for the rest of the procedure, and the rf voltage $U_{1}$ of the $28 \mathrm{MHz}$ system is ramped up in a stepwise manner from 192 to $300 \mathrm{kV}$, where it also remains for the rest of the procedure.

This procedure leads to a rotation of the bunch in longitudinal phase space, effectively shortening the bunch

TABLE I. rf parameter set points for the rebucketing procedure. Wall current monitor data taking starts at turn 0 .

\begin{tabular}{rcccc}
\hline \hline \multicolumn{1}{c}{ Turn } & $U_{1} / \mathrm{kV}$ & $\psi_{1}$ & $U_{2} / \mathrm{kV}$ & $\psi_{2}$ \\
\hline 0 & 192 & $\pi$ & 0.0 & $\pi$ \\
890 & 192 & $\pi$ & 0.0 & $\pi$ \\
891 & 192 & 0.0 & 0.0 & $\pi$ \\
1324 & 192 & 0.0 & 0.0 & $\pi$ \\
1325 & 300 & $\pi$ & 0.0 & $\pi$ \\
2193 & 300 & $\pi$ & 0.0 & $\pi$ \\
2194 & 300 & $\pi$ & 750 & $\pi$ \\
2302 & 300 & $\pi$ & 750 & $\pi$ \\
2303 & 300 & $\pi$ & 1500 & $\pi$ \\
$\vdots$ & $\vdots$ & $\vdots$ & $\vdots$ & $\vdots$ \\
80497 & 300 & $\pi$ & 1900 & $\pi$ \\
\hline \hline
\end{tabular}




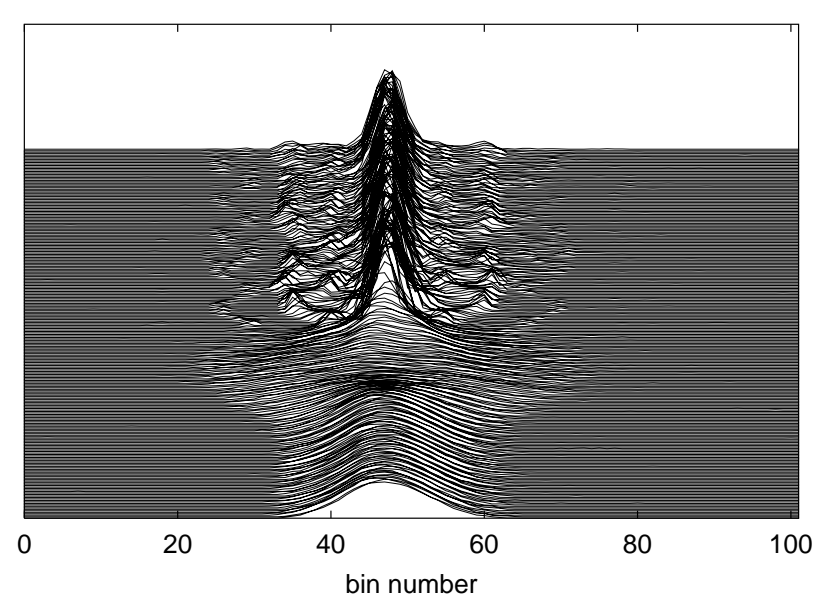

FIG. 3. Mountain range plot comprising 250 profiles obtained using the wall current monitor during the rebucketing of fill 01484 in the RHIC Blue ring. Profiles were acquired every 20 turns.

length. To capture the beam and preserve this short bunch length, the $197 \mathrm{MHz}$ storage rf system voltage $U_{2}$ is turned on in two steps, first to $750 \mathrm{kV}$ at turn 2194 , and then to $1500 \mathrm{kV}$ at turn 2303. From there it is slowly ramped within $3 \mathrm{sec}$ to its final voltage of $U_{2}=2700 \mathrm{kV}$, which corresponds to $U_{2}=1900 \mathrm{kV}$ after $1 \mathrm{sec}$, or 78195 turns. Figure 3 shows a mountain range of 250 profiles obtained during rebucketing of fill 01484 in the RHIC Blue ring.

Taking these input data, a tomographic reconstruction of the 2-dimensional longitudinal phase space distribution was attempted. For a successful reconstruction several parameters had to be adjusted by a fitting procedure: (i) As already mentioned earlier, the time between the beginning of data taking and the actual start of the rebucketing process varies from fill to fill. (ii) The position of the synchronous phase within the profiles does not coincide with the center bin of the measured profiles, as Fig. 3 shows.

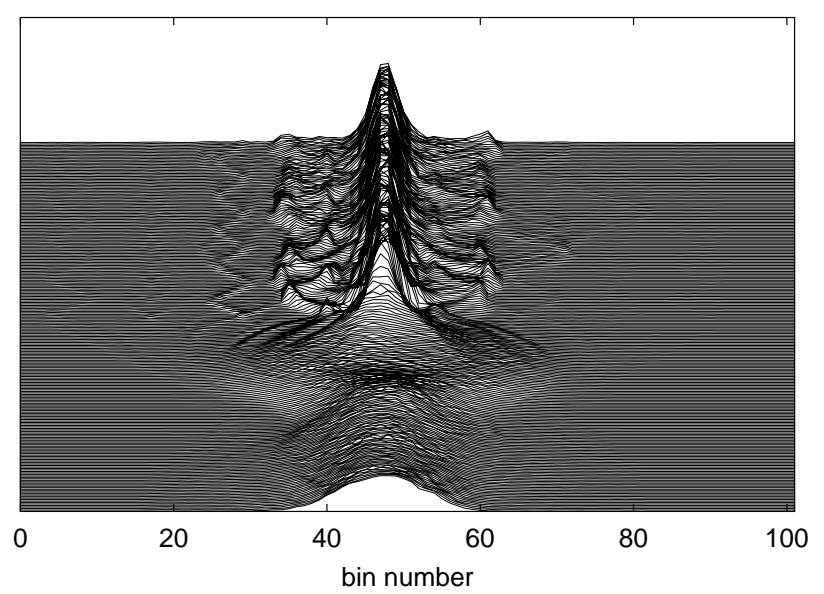

FIG. 4. Mountain range plot of 250 profiles, as obtained from the reconstructed phase space distribution. The time between profiles corresponds to 20 revolutions in the RHIC.
TABLE II. Measured rf parameter set points for the rebucketing procedure of fill 01484 in the RHIC Blue ring, as found by a fitting procedure. Wall current monitor data taking starts at turn 0. Compare to Table I.

\begin{tabular}{rccrc}
\hline \hline Turn & $U_{1} / \mathrm{kV}$ & $\psi_{1}$ & $U_{2} / \mathrm{kV}$ & $\psi_{2}$ \\
\hline 0 & 203.9 & $\pi$ & 0.47 & 0.753 \\
1105 & 203.9 & $\pi$ & 0.47 & 0.753 \\
1106 & 244.9 & -0.273 & 0.47 & 0.753 \\
1539 & 244.9 & -0.273 & 0.47 & 0.753 \\
1540 & 318.7 & $\pi$ & 0.47 & 0.753 \\
2408 & 318.7 & $\pi$ & 0.47 & 0.753 \\
2409 & 318.7 & $\pi$ & 534.7 & 3.87 \\
2517 & 318.7 & $\pi$ & 534.7 & 3.87 \\
2518 & 318.7 & $\pi$ & 1831.3 & 3.53 \\
2625 & 318.7 & $\pi$ & 1831.3 & 3.53 \\
2626 & 318.7 & $\pi$ & 1831.9 & 3.53 \\
2634 & 318.7 & $\pi$ & 1831.9 & 3.53 \\
$\vdots$ & $\vdots$ & $\vdots$ & $\vdots$ & $\vdots$ \\
\hline \hline
\end{tabular}

(iii) The voltages of both rf systems as seen by the beam may differ from the set points. (iv) The zero voltage of the $197 \mathrm{MHz}$ rf system is achieved by counterphasing three cavities with nominal voltages of $10 \mathrm{kV}$ each. This may result in a nonzero voltage and an arbitrary phase. (v) The voltage of the $197 \mathrm{MHz}$ rf system may not behave linearly when ramped up quickly. (vi) The $197 \mathrm{MHz}$ rf system may not be exactly in phase with the $28 \mathrm{MHz}$ cavities.

Taking these uncertainties as free parameters, a simulated annealing algorithm [4] was used to minimize the discrepancy $\Delta$ between measured and reconstructed profiles as given by Eq. (3). Figure 4 displays a mountain range of profiles obtained from the reconstructed phase space distribution, which was calculated using the optimized parameters given in Table II. Using the nominal parameters given in Table I but with the correct timing and

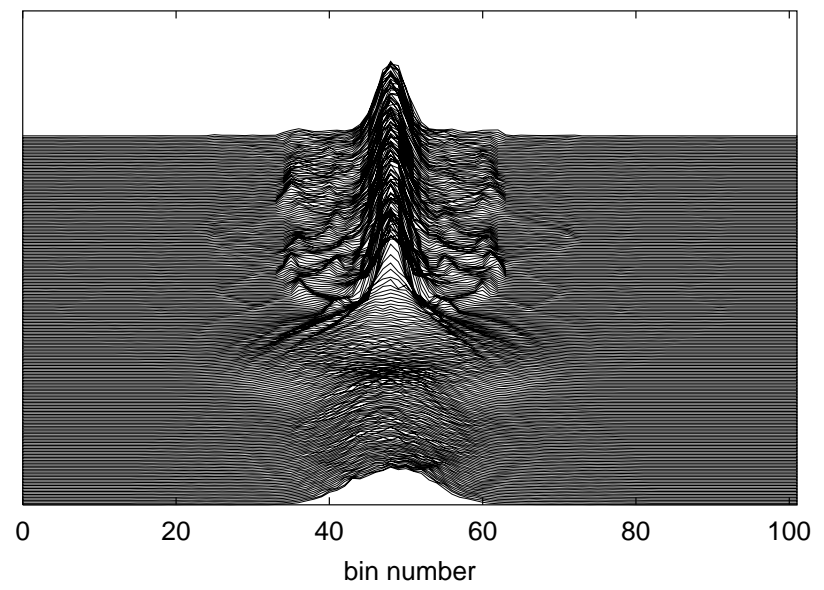

FIG. 5. Mountain range plot of 250 profiles, obtained from a reconstructed phase space distribution using nominal machine parameters. The time between profiles correponds to 20 revolutions of the beam in the RHIC. 

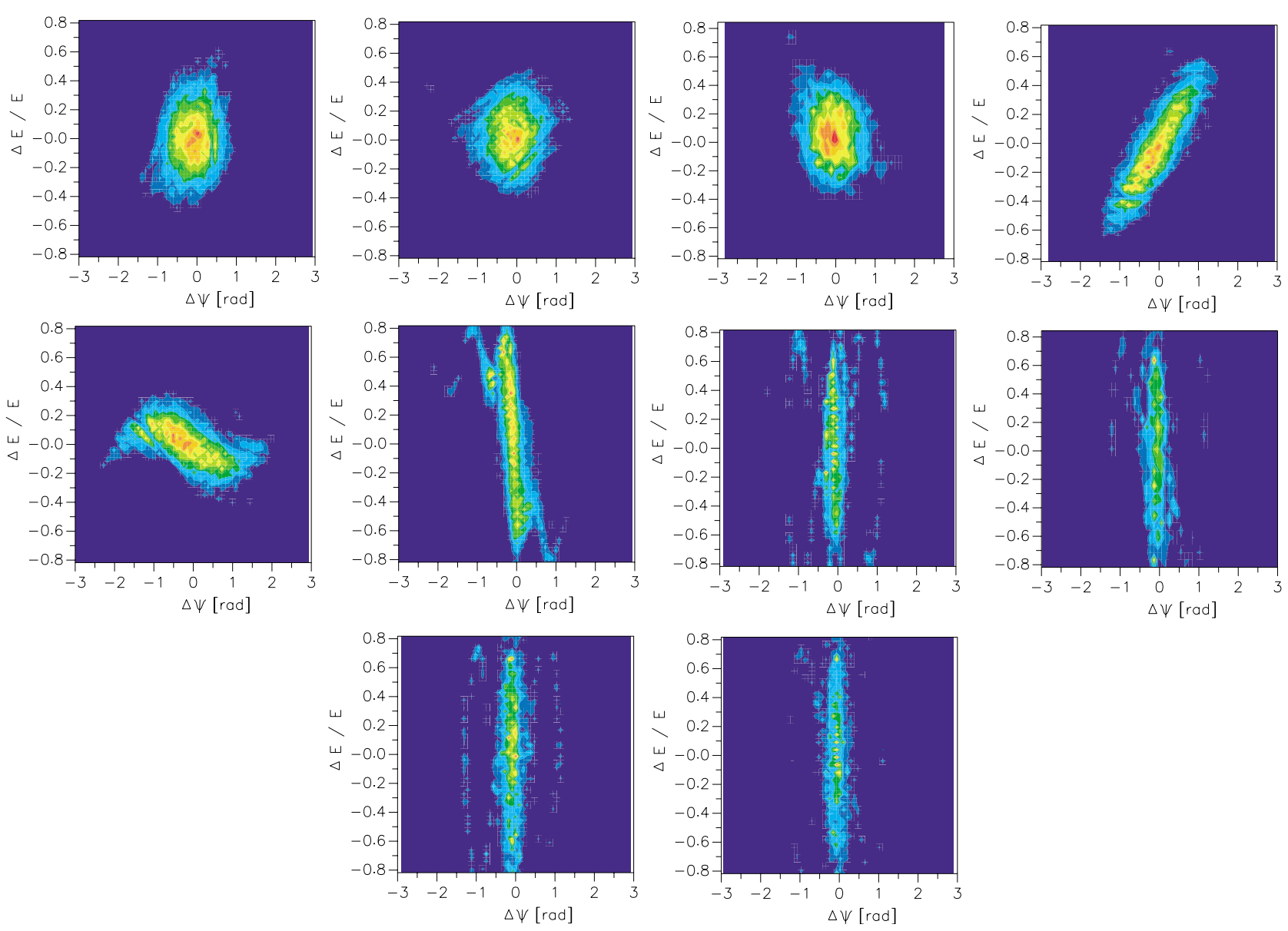

FIG. 6. (Color) Evolution of the phase space distribution during rebucketing. Reconstructions are performed at each 25th profile.

synchronous phase position for the reconstruction results in a significantly worse agreement between measured and reconstructed profiles. The remaining discrepancy $\Delta$ after 50 iterations is about $66.5 \%$ higher than in the case with optimized parameters, though longitudinal bunch profiles obtained from this reconstruction agree well with measured data at first glance; see Fig. 5.

Using longitudinal profiles as obtained from the reconstructed distribution as input data, the parameter fitting algorithm was tested. While the actual parameters used for the reconstruction were the optimized ones, as given in Table II, the fitting started at the nominal parameter set given in Table I. The correct rf parameters could be determined within an accuracy of $\pm 2 \%$. Additionally, this test also revealed that in the case of real data the remaining discrepancy for optimized parameters is dominated by measurement noise. When (noise-free) profiles from the reconstructed distribution are used, the remaining discrepancy after 50 iterations is smaller than the corresponding value for measured data by a factor of 3 . Having successfully reconstructed the phase space distribution before rebucketing, it is straightforward and instructive to show its evolution during the bunch rotation process. This is depicted in Fig. 6.

\section{CONCLUSION}

For the first time, tomographic reconstruction has been successfully applied to bunch rebucketing when frequent rf parameter changes occur. Since the technique affords a quantitative measure of the reconstruction quality, several of these rf parameters were effectively measured during the rebucketing process.

\section{ACKNOWLEDGMENTS}

We would like to thank S. Peggs and T. Satogata for encouraging discussions, and S. Hancock and M. Lindroos for providing the parallelized version of their tomography code. This work was performed under the auspices of the U.S. Department of Energy.

[1] S. Hancock, M. Lindroos, and S. Koscielniak, Phys. Rev. ST Accel. Beams 3, 124202 (2000).

[2] C. Montag et al., in Proceedings of the EPAC 2002, Paris.

[3] C. Montag et al., Phys. Rev. ST Accel. Beams (to be published).

[4] W.H. Press et al., Numerical Recipes in C: The Art of Scientific Computing (Cambridge University Press, Cambridge, 1992), 2nd ed. 